\title{
A CMOS MEMS Thermal Sensor with High Frequency Output
}

\author{
S.-H. Tseng ${ }^{1,2}$, C.-L. Fang ${ }^{2}$, P.-C. Wu ${ }^{2}$, Y.-Z. Juang ${ }^{2}$, and Michael S.-C. Lu ${ }^{1}$ \\ ${ }^{1}$ Institute of Electronics Engineering, National Tsing Hua University, Hsinchu, Taiwan, R.O.C. \\ ${ }^{2}$ Chip Implementation Center, National Applied Research Laboratories, Hsinchu, Taiwan, R.O.C. \\ memsshtseng@gmail.com
}

\begin{abstract}
This work presents the design and characterization of a CMOS-integrated thermal sensor that features a novel oscillator-based sensing interface to achieve a high thermoelectric sensitivity. The thirty pairs of thermocouples are made of $\mathrm{n}^{+} / \mathrm{p}^{+}$polysilicon of a standard $0.18-\mu \mathrm{m}$ CMOS to produce a large thermoelectric voltage. The produced thermoelectric voltage is used to control the bias current of a high-frequency oscillator circuit and causes a shift in the output frequency. Experimental result indicates the $544-\mathrm{MHz}$ oscillator has a temperature sensitivity of $46.3 \mathrm{kHz} /{ }^{\circ} \mathrm{C}$.
\end{abstract}

\section{INTRODUCTION}

Thermopile-type thermal and infrared sensors are used in a number of applications, including inertial sensing [1][2], low-cost mass-produced infrared thermal imagers [3][4], medical thermograph, and security monitoring devices. Thermoelectric sensors have the advantage of producing a linear self-generated response without the need of temperature stabilization and cooling operation. The linear thermoelectric signal transduction and the wide sensing range make it attractive for many low-cost commercial applications. The thermopile can directly convert thermal signals like temperature or heat to electrical signals. With the integration of the low-noise CMOS instrumentation amplifier and chopper technique, the offset and low frequency noise can be suppressed to avoid the disturbance on the thermoelectric signals [5].

Thermal microsensors based on CMOS technology become feasible when the CMOS-compatible micromachining process is established. The micromachining process can remove the thermally conductive silicon substrate to provide a better isolation for enhancing the signal-to-noise ratio. Prior work has reported thermal sensors utilizing materials in a CMOS process to fabricate thermopiles [3][6]. Schaufelbuhl [3] reported a thermoelectric CMOS infrared sensor array composed of polysilicon/aluminum thermopiles with a combined Seebeck coefficient of $108 \mu \mathrm{V} / \mathrm{K}$. Akin [7] utilized the n-poly/p $\mathrm{p}^{+}$ diffusion to fabricate thermocouples with a higher combined
Seebeck coefficient of $750 \mu \mathrm{V} / \mathrm{K}$. The most effective combination for raising the Seebeck coefficient is to connect the p-type and n-type polysilicon directly without the via metal in CMOS process. Our approach uses a standard 0.18$\mu \mathrm{m}$ CMOS followed by a post micromachining process to fabricate suspended thermopiles made of $\mathrm{n}^{+} / \mathrm{p}^{+}$polysilicon and takes advantage of the fast electronics to implement a high-frequency oscillator circuit for enhancing the sensor sensitivity. This idea has the potential to make highperformance thermopile-based infrared sensors in the future.

\section{DEVICE FABRICATION}

The TSMC $0.18-\mu \mathrm{m}$ one-polysilicon six-metal (1P6M) CMOS process is used for device fabrication. Fig. 1 shows the micrograph of the thermal sensor consisting of the thermopiles and the differential ring oscillator circuit. The process flow in Fig. 2 shows the development of the sensor. As shown in Fig. 2(a), the passivation layers on etch selective regions are removed during the CMOS process to facilitate the following oxide etch. A thick photoresist layer is spun and patterned after completion of CMOS to protect the bond pads and circuit regions from the oxide etch. The oxide layers are removed anisotropically by a reactive ion etch using $\mathrm{CH}_{4}, \mathrm{CHF}_{3}$ and $\mathrm{O}_{2}$ as shown in Fig. 2(b). This step removes the oxide between the meandering thermopile structures. In Fig. 2(c), an isotropic dry etch using $\mathrm{SF}_{6}$ and $\mathrm{O}_{2}$ is performed to remove the silicon between the hot and the cold regions of polysilicon for reducing thermal conduction through the substrate. The foregoing post-CMOS micromachining steps have been verified previously for fabrication of a CMOS-compatible MEMS VCO [8]. The measured results show good reliability and compatibility with the standard CMOS process. 


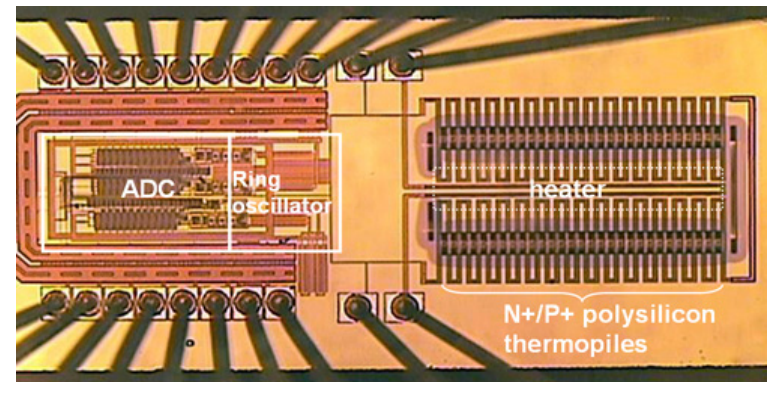

Figure 1. Micrograph of the thermal sensor consisting of the thermopile and the VCO circuit.
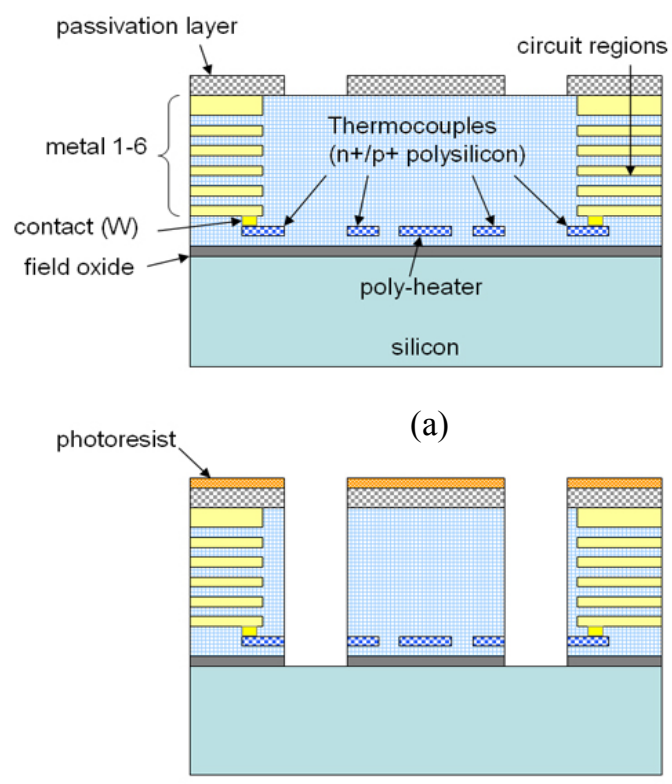

(b)

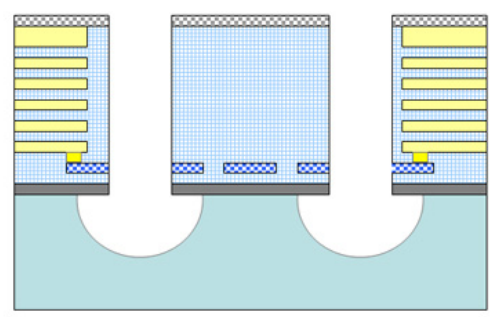

(c)

Figure 2. Post-CMOS micromachining steps: (a) after completion of CMOS, (b) anisotropic oxide etch, and (c) isotropic etch to remove silicon substrate.

\section{DESIGN}

The released thermopile microstructure as shown in Fig. 3 consists of alternating $\mathrm{n}^{+}$and $\mathrm{p}^{+}$polysilicon layers for thermal sensing. The respective doping processes are accomplished by the CMOS foundry. The combination of a n-type and a p-type polysilicon layers, due to their large and opposite Seebeck coefficients [9], provides a large thermoelectric voltage that can be processed by the sensing circuit. The heater and the thirty-pair thermopiles occupy an area of $650 \mu \mathrm{m}$ by $440 \mu \mathrm{m}$. The polysilicon heater has a resistance of $0.9 \mathrm{k} \Omega$ and is placed in the center of the thermopiles for producing the desired temperature change during test.

Fig. 4 shows the differential ring oscillator circuit for thermoelectric sensing, in which the produced thermoelectric voltage changes the control voltage $\mathrm{Vc}$ of the bias circuit and causes an output frequency shift accordingly. The delay in the cell is decided by the charge at each node and the current through the load. The load can normally use a resistor for a fixed oscillation frequency, or PMOS transistors for making a tunable oscillator. The PMOS load is usually implemented in a symmetric or cross-coupled topology. The frequency of the ring oscillator with a differential topology can be expressed by eq. (1),

$$
f_{c}=\frac{1}{2 \times N \times t_{d}} \cong \frac{I_{\text {tail }}}{2 \times N \times V_{\text {swing }} \times C_{\text {node }}}
$$

where $\mathrm{N}$ is the number of stages used, $\mathrm{t}_{\mathrm{d}}$ is the stage delay, $\mathrm{V}_{\text {swing }}$ is the single-ended swing on the drain, $\mathrm{C}_{\text {node }}$ is the total capacitance on the differential stage output node, and $\mathrm{I}_{\text {tail }}$ is the tail current [10].

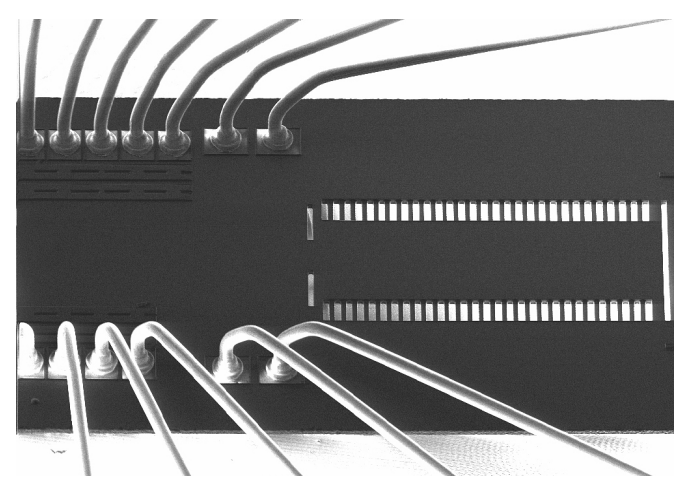

Figure 3. Scanning electron micrograph of the released thermopiles after wirebond. 

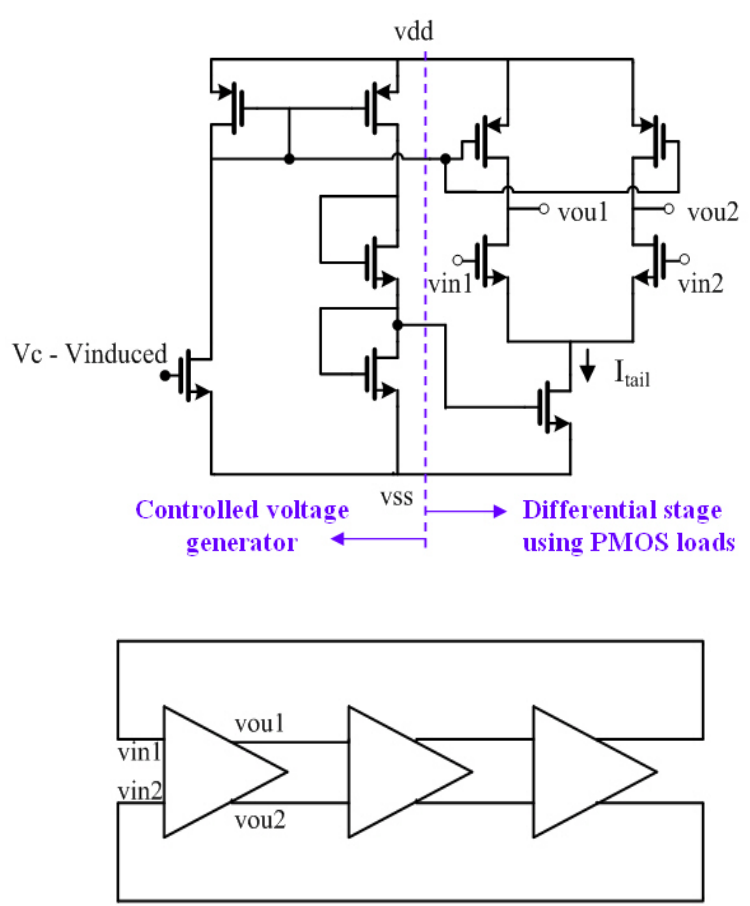

Figure 4. Schematic of the differential ring oscillator with its output frequency controlled by the thermoelectric voltage.

\section{EXPERIMENT}

As shown in Fig. 5, the temperature distribution of the heated microstructure with an applied heating voltage of $11 \mathrm{~V}$ was obtained by infrared measurement (InfraScope II). The whole chip was pre-heated close to about $75^{\circ} \mathrm{C}$ before measurement for enhancing the accuracy. The temperature of the heater was gradually increased by the applied voltage, and the maximum temperature occurred at the corner of the heater. Fig.6 shows the maximum temperature with respect to the applied power of the polysilicon heater.

The oscillating frequency shown in Fig. 7 was measured by a spectrum analyzer (Agilent-E4440A) with a control voltage $\mathrm{V}_{\mathrm{c}}$ varied between 0.68 to $0.96 \mathrm{~V}$. The frequencytuning capability is about $600 \mathrm{MHz} / \mathrm{V}$. Next the heating voltage was applied to produce the thermoelectric voltage which was in series with a fixed $V_{c}$ of $0.96 \mathrm{~V}$. The output spectra plotted in Fig. 8 were measured for heating voltages of $0 \mathrm{~V}$ and $30 \mathrm{~V}$, respectively.

The oscillating frequency changes from 544 to $526 \mathrm{MHz}$ when the heating voltage increases from $0 \mathrm{~V}$ to $30 \mathrm{~V}$. The corresponding temperature increases from $75^{\circ} \mathrm{C}$ to $464^{\circ} \mathrm{C}$. Fig. 9 shows the relationship of the measured frequency with respect to the maximum heater temperature. The temperature sensitivity in terms of the frequency shift is $46.3 \mathrm{kHz} /{ }^{\circ} \mathrm{C}$.

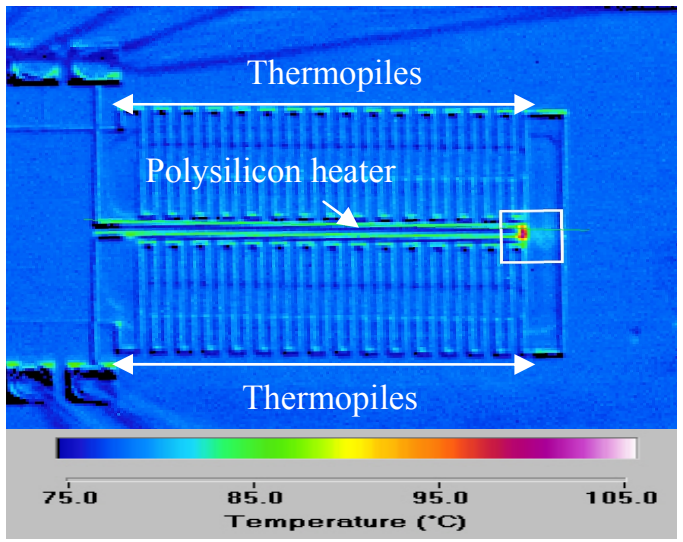

Figure 5. Measured temperature distribution of the polysilicon heater.

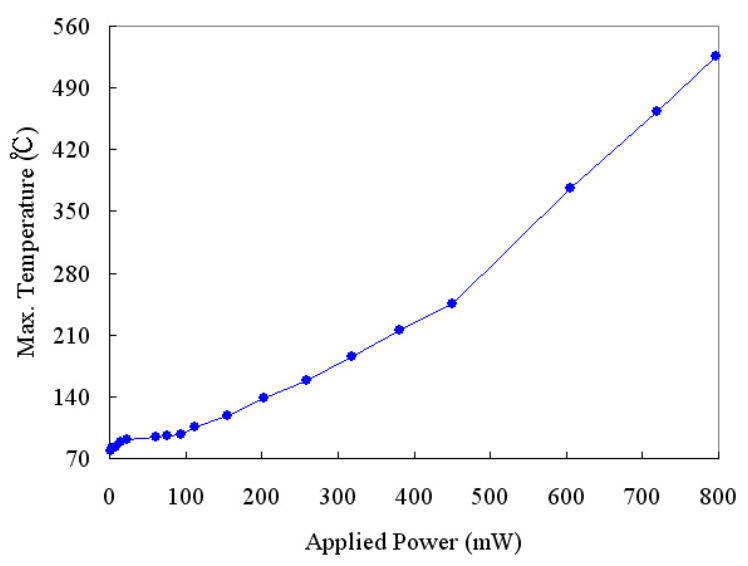

Figure 6. Measured temperature vs. applied power.

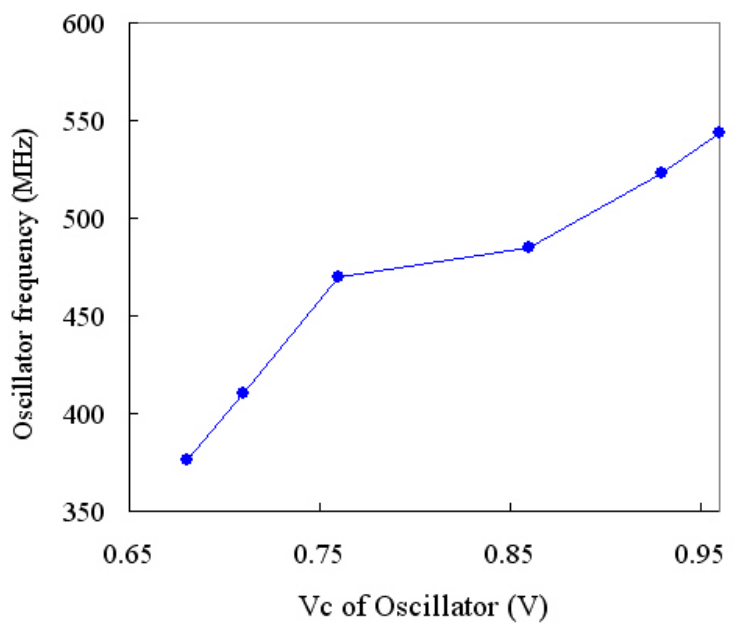

Figure 7. Relationship of the measured oscillating frequency with respect to the control voltage $V_{c}$. 


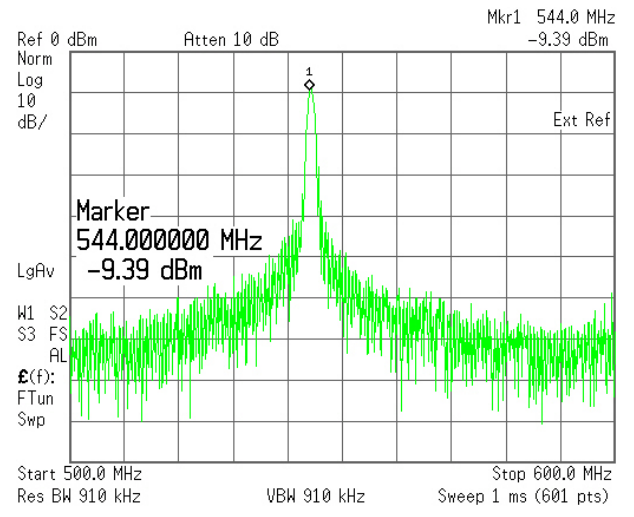

(a)

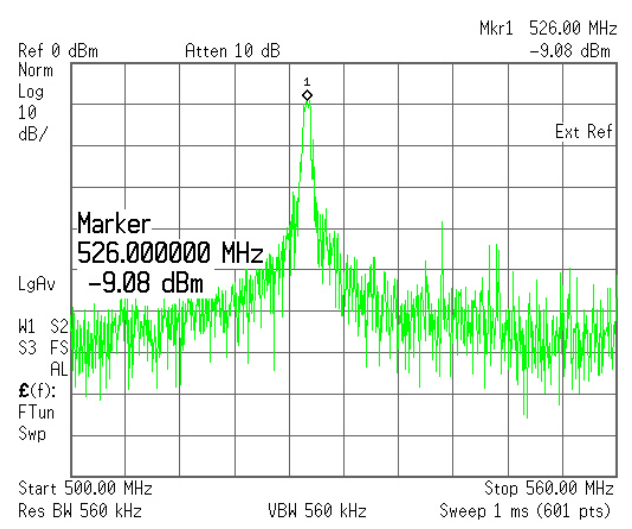

(b)

Figure 8. Measured spectrum at (a) heating bias $=0 \mathrm{~V}$; and $(b)$ heating bias $=30 \mathrm{~V}$.

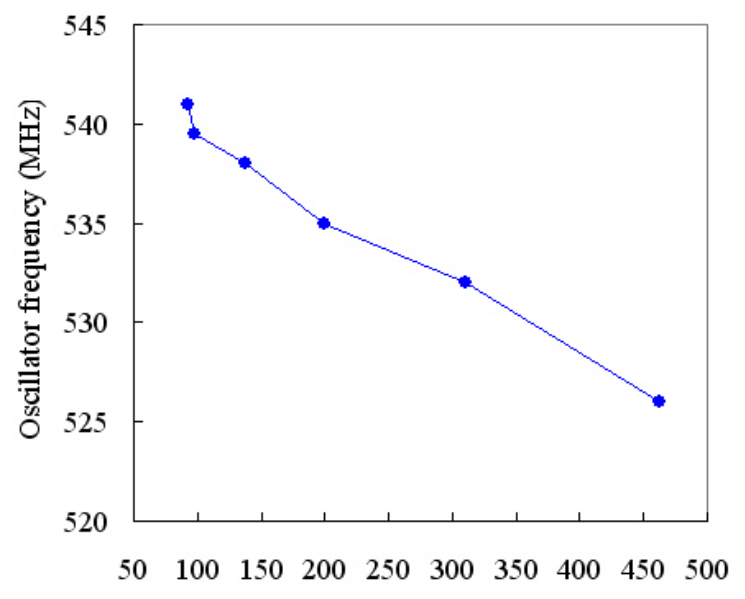

Maximum temperature at polysilicon heater $\left({ }^{\circ} \mathrm{C}\right)$

Figure 9. Oscillating frequency output vs. maximum heater temperature.

\section{CONCLUSION}

In this paper, we have proposed a novel oscillator-based sensing interface to achieve a high thermoelectric sensitivity. The suspended microstructures with $\mathrm{n}^{+}$and $\mathrm{p}^{+}$polysilicon layers can be successfully fabricated through the CMOS micromachining steps. To further enhance the sensitivity, the hot points of the thermopiles can be placed in a more concentrated region to have a better heating efficiency, and the oscillating frequency of the ring oscillator can be further raised to a higher frequency such as $\mathrm{GHz}$.

\section{ACKNOWLEDGMENT}

The authors would like to thank the National Chip Implementation Center for support of the research and the chip fabrication and Professor Da-Jeng Yao for support of the laboratory resources.

\section{REFERENCES}

[1] D. Goustouridias, G. Kaltsas, A. G. Nassiopoulou, "A silicon thermal accelerometer without solid proof mass using porous silicon thermal isolation," IEEE Journal of Sensors, vol. 7, no. 7, 2007, pp. 983-989..

[2] S. H. Tsang, A. H. Ma, K. S. Karim, A. Parameswaran, and A. M. Leung, "Monolithically fabricated polymermems 3-axis thermal accelerometers design for automated wirebonder assembly," IEEE MEMS Conf., 2008, pp. 880-883.

[3] A. Schaufelbuhl, N. Schneeberger, U. Munch, M. Waelti, O. Paul, O. Brand, H. Baltes, C. Menolfi, Q. Huang, E. Doering, M. Loepfe, "Uncooled low-cost thermal imager based on micromachined CMOS integrated sensor array," IEEE J. Microelectromechanical Systems, vol. 10, no. 40, 2001, pp. 503-510.

[4] H. Baltes, and O. Brand, "CMOS-based microsensors and packaging," Sensors and Actuators A, vol. 92, 2001, pp. 1-9.

[5] C. Menolfi, and Q. Huang, "A low-noise CMOS instrumentation amplifier for thermoelectric infrared detectors," IEEE J. Solid-State Circuits, vol. 32, no. 7, 1997, pp. 968-976.

[6] U. Munch, D. Jaeggi, K. Schneeberger, A. Schaufelbuhl, O. Paul, H. Baltes, and J. Jasper, "Inductrial fabrication technology for CMOS infrared sensor arrays," Transducers Conf., pp. 205-208, 1997.

[7] T. Akin, Z. Olgun, O. Akar, H. Kulah, " An integrated thermopile structure with high responsivity using any standard CMOS process," Sensors and Actuators A, vol. 66, 1998, pp.218-224.

[8] S. H. Tseng, Y. J Huang, Y. Z. Juang, and M. S.-C. Lu, "A 5.8-GHz VCO with CMOS-compatible MEMS inductors," Sensors and Actuators A, vol. 139, 2007, pp. 187-193.

[9] S. M. Sze, Semiconductor Sensors, John Wiley and Sons, New York, 1994.

[10] A. Hajimiri, T.-H. Lee, Low Noise Oscillators, Kluwer Academic Publishers, Boston, 1999. 